\title{
NOVOS CRITÉRIOS DIAGNÓSTICOS DE SEPSE E SUA APLICAÇÃO NO PRONTO ATENDIMENTO HOSPITALAR
}

\section{ARTIGO DE REVISÃO}

TORRES, Fernanda Rodrigues ${ }^{1}$

RODRIGUES, Simone Alves Pereira ${ }^{2}$

SILVA, Fabiana Martins $\mathrm{da}^{3}$

NUEVO, Ricardo Antonio Bottura ${ }^{4}$

TORRES, Fernanda Rodrigues. Et al. Novos critérios diagnósticos de sepse e sua aplicação no pronto atendimento hospitalar. Revista Científica Multidisciplinar

1 Mestre em Biotecnologia pela Universidade de Ribeirão Preto - UNAERP; Especialista em Educação Profissional na área de Saúde - Enfermagem pela Fundação Osvaldo Cruz - FIOCRUZ; Especialista em Centro Cirúrgico pela Universidade de Ribeirão Preto - UNAERP; Graduação em Enfermagem e Obstetrícia pela Universidade do Estado de Minas Gerais - UEMG; Acadêmica de Medicina pela Universidade Brasil.

${ }^{2}$ Especialista em Prótese Dentária pela Faculdade São Leopoldo Mandic; Graduação em Odontologia pela FOA- Faculdade de Odontologia de Araçatuba- UNESP; Acadêmica de Medicina pela Universidade Brasil.

${ }^{3}$ Especialização em Farmacologia pelo Conselho Regional de Farmácia - CRF-MS; Pós-Graduação em Gestão de Assistência Farmacêutica pela Universidade Federal do Mato Grosso do Sul - UFMS; Graduação em Farmácia Bioquímica pela Universidade para o Desenvolvimento do Estado e da Região do Pantanal UNIDERP; Acadêmica de Medicina pela Universidade Brasil.

${ }^{4}$ Especialista em Anestesiologia pela Sociedade Brasileira de Anestesiologia - SBA, Especialista em Dor pela Sociedade Brasileira de Anestesiologia - SBA, Especialista em Medicina Legal pela Polícia Civil do Estado de São Paulo. 
Núcleo do Conhecimento. Ano 04, Ed. 06, Vol. 09, pp. 96-104. Junho de 2019. ISSN: 2448-0959

\section{RESUMO}

A Sepse é considerada um grande desafio diagnóstico devido à necessidade de seu reconhecimento imediato bem como de seu tratamento precoce. Sua alta taxa de mortalidade está relacionada à demora do diagnóstico bem como com a inadequação da abordagem do agente infeccioso. O objetivo deste trabalho é descrever os novos critérios diagnósticos de sepse aplicados na prática de pronto atendimento hospitalar para evitar futuras complicações assim como os altos índices de mortalidade. Tratase de um estudo de pesquisa exploratório e descritivo, ancorado nas diretrizes da Society of Critical Care Medicine (SCCM) e da European Society of Critical Care Medicine (ESICM) 2016 que discorrem sobre o novo consenso internacional a partir de critérios diagnósticos para a sepse e o choque séptico. Até o momento foram realizadas três grandes conferências para atualização dos conceitos de sepse: a Sepsis-1 (1992), Sepsis-2 (2001) e Sepsis-3 (2016). Essas conferências tiveram como objetivo padronizar as definições e termos relacionados a sepse, a tratando como uma forma de dinamizar e agilizar o processo de diagnóstico e tratamento. O termo "sepse grave" foi abolido uma vez que todos os casos de sepse devem ser vistos como graves, sendo seu diagnóstico clínico não mais baseado nos critérios da SIRS e sim no preenchimento de 2 ou mais pontos no escore SOFA. Este novo score passou a considerar variáveis como a elevação da frequência respiratória e a diminuição do nível de consciência. Concluiu-se que para a prática dos novos critérios é necessária atualização dos profissionais de saúde com a finalidade de identificar e tratar a sepse em tempo oportuno, garantindo, assim, melhores resultados bem como a diminuição dos óbitos.

Palavras-chave: Sepse, critérios diagnósticos, atendimento hospitalar. 


\section{INTRODUÇÃO}

O organismo frente a uma situação infecciosa seja esse causada por vírus, bactérias, fungos ou mesmo protozoários definiu-se como Sepse. Devido aos diferentes tipos de manifestações e seus estágios clínicos, esta afecção se trata de um desafio para médicos de quase todas as especialidades, por conta da necessidade de um pronto reconhecimento e do tratamento precoce (BARACHO et al, 2011). Estudos evidenciaram índices elevados da sepse nas UTIs do Brasil em termos de mortalidade, tendo o país uma das taxas mais elevadas do mundo todo. Dessa forma, os pacientes possuem quadros mais graves e precisam de um tempo maior de internação. No começo do presente século, ainda se buscava uma compreensão melhor acerca da doença, devido à falta de dados. Avanços em relação ao diagnóstico precoce, ao rastreamento microbiano, ao uso mais otimizado das variáveis hemodinâmicas e das técnicas de suporte orgânico tem evoluído no campo das pesquisas científicas (JUNIOR, et al 2006).

A alta mortalidade por sepse nas fases mais avançadas está relacionada à inadequação da abordagem do agente infeccioso. O controle do foco infecioso é primordial para que a antibioticoterapia tenha sucesso na eliminação do agente agressor. A escolha inicial inadequada do esquema antimicrobiano pode levar ao aumento significativo da taxa de mortalidade em pacientes sépticos (SATRIANO, 2017). Conforme o consenso de 2008, sepse, sepse grave ou choque séptico representam a evolução temporal da mesma síndrome, associado às taxas crescente de mortalidade e de gravidade. Quanto mais tempo se demorar para realizar o diagnóstico, maior a chance de o quadro tornar-se grave, aumentando, assim, a mortalidade (CARNEIRO; PÓVOA; GOMES, 2017). A ampliação dos conhecimentos no campo da saúde traz inovações ao diagnóstico e à terapêutica, transformando-se em temas de investigação científica.

Pode-se enfatizar o recente papel das técnicas proteômicas, sobretudo na identificação de todas as proteínas codificadas no genoma têm adquirido no estudo da sepse, em termos da fisiopatologia, do diagnóstico, da terapêutica e do prognóstico para a incorporação à prática clínica (BATISTA, et al, 2012). Foi promovido, 
recentemente, pela Society of Critical Care Medicine (SCCM) e pela European Society of Critical Care Medicine (ESICM) uma nova conferência para a publicação de novas definições relacionadas à sepse, conhecida como Sepsis 3. Essa definição ampla de sepse pela nova publicação é definida pela "presença de disfunção orgânica ameaçadora à vida secundária à resposta desregulada do organismo à infecção", sendo o diagnóstico clínico de disfunção orgânica baseado na variação de dois ou mais pontos no escore Sequential Organ Failure Assessment (SOFA) (MOURA ET $A L, 2017)$. A presença dos critérios da síndrome da resposta inflamatória sistêmica (SRIS) não é mais necessária para a definição.

Todos os casos de sepse devem ser considerados como uma doença grave, abolindo, assim, a expressão "sepse grave". Nesse sentido, define-se o choque séptico como "um subgrupo dos pacientes com sepse que apresentam acentuadas anormalidades circulatórias, celulares e metabólicas e associadas com maior risco de morte do que a sepse isoladamente". É necessário nos critérios diagnósticos de choque séptico observar-se a "necessidade de vasopressor para manter uma pressão arterial média acima de $65 \mathrm{mmHg}$ após a infusão adequada de fluidos, associada a nível sérico de lactato acima de $2 \mathrm{mmol} / \mathrm{L}$ " (MACHADO, et al, 2016). Estudos mostram contradições médicas frente ao novo conceito de identificação da Sepse seja em qualquer área hospitalar. As críticas concentram-se em três aspetos: quanto aos conceitos teóricos que os informam, quanto a metodologia seguida e quanto ao seu potencial impacto na aplicação clínica (CARNEIRO; PÓVOA; GOMES, 2017).

\section{DESENVOLVIMENTO}

\subsection{METODOLOGIA}

Trata-se de estudo de pesquisa exploratória e descritiva com o objetivo de buscar informações junto a revisão narrativa da literatura, ancorada nas diretrizes da Society of Critical Care Medicine (SCCM) e da European Society of Critical Care Medicine (ESICM) 2016 sobre o novo consenso internacional sobre os critérios diagnósticos para sepse e choque séptico, obtendo-se, nesse sentido, uma nova percepção dele, visto que houve a descoberta de ideias novas a partir da observação, registro, análise 
e correlação de fatos elaborados por meio da pesquisa bibliográfica. Como instrumento de coleta de informações, foi realizada uma pesquisa bibliográfica em livros, revistas e outros textos bem como consultas a sites da internet para maior embasamento teórico sobre o assunto abordado neste trabalho. Foi feita uma abordagem qualitativa do tema por considerar-se um assunto complexo e que envolve vários aspectos que interferem na realidade dos processos aplicativos da prática sobre o diagnóstico de sepse no pronto atendimento hospitalar.

\subsection{OBJETIVO GERAL}

Descrever os novos critérios diagnósticos de sepse aplicados na prática de pronto atendimento hospitalar para evitar complicações futuras e altos índices de mortalidade.

\subsection{DISCUSSÃO}

O crescente aumento da incidência da sepse exigiu na adoção de medidas urgentes para seu enfrentamento em decorrência das complicações trazidas aos pacientes bem como para a saúde pública, do ponto de vista coletivo. A população idosa vem crescendo assim como o número de pacientes imunossuprimidos ou portadores de doenças crônicas. Tais fatores facilitam o desenvolvimento de infecções graves (ILAS, 2015). Além disso, a maior resistência à microorganismos e a falta de infraestrutura de atendimento em prontos-socorros e hospitais facilitam a sua disseminação. Levantam-se estimativas que apontam a existência de aproximadamente 600 mil novos casos de sepse a cada ano no Brasil. Este cenário tem impacto direto nos indicadores de morbi-mortalidade, sendo que as consequências da sepse são responsáveis pelas causas de $16,5 \%$ dos atestados de óbitos emitidos, ou seja, em torno de 250 mil casos, tornando-se um grave desafio para a saúde pública (ILAS, 2015).

Sepse é uma doença dinâmica que está entre as principais causas de morte em UTI sendo um grande desafio a ser vencido (JUNIOR, et al 2006). Segundo Shorr (2007), as principais definições estão relacionadas à síndrome de resposta inflamatória 
sistêmica, sepse, sepse grave e choque séptico. Na síndrome da resposta inflamatória sistêmica (SRIS) há a presença de pelo menos 2 dos seguintes itens:

a) temperatura central $>38,3^{\circ} \mathrm{C}$ ou $<36^{\circ} \mathrm{C}$;

b) frequência cardíaca > 90bpm;

c) frequência respiratória $>20 \mathrm{rpm}$ ou $\mathrm{PaCO} 2<32 \mathrm{mmHg}$ ou necessidade de ventilação mecânica;

d) leucócitos totais $>12.000 / \mathrm{mm}^{3}$ ou $<4.000 / \mathrm{mm}^{3}$ ou presença de $>10 \%$ de formas jovens (SHORR, 2007).

A Sepse é secundária ao processo infeccioso confirmado ou suspeito, sem necessidade da identificação do agente infeccioso (SHORR, 2007). Já a Sepse grave contém a presença dos critérios de sepse associada à disfunção orgânica ou sinais de hipoperfusão. Hipoperfusão e anormalidades da perfusão podem incluir, mas não estão limitadas a elementos como a hipotensão, hipoxemia, acidose láctica, oligúria e alteração aguda do estado mental (SHORR, 2007). O choque séptico, por sua vez, é um estado de falência circulatória aguda caracterizada pela persistência de hipotensão arterial em paciente séptico, sendo a hipotensão definida como a pressão arterial sistólica $<90 \mathrm{mmHg}$, redução de $>40 \mathrm{mmHg}$ da linha de base, ou pressão arterial média $<60 \mathrm{mmHg}$, a despeito de adequada reposição volêmica, com necessidade de vasopressores, na ausência de outras causas de hipotensão (SHORR, 2007).

A Síndrome da Resposta Inflamatória Sistêmica (SIRS), tem como conceito uma particularidade clínica, não sendo necessário a causa por uma infecção. A SIRS se caracteriza pelos parâmetros de: temperatura maior que $38^{\circ} \mathrm{C}$ ou menor que $36^{\circ} \mathrm{C}$, frequência cardíaca maior que $90 \mathrm{bpm}$, frequência respiratória superior a 20/respirações por minuto ou hiperventilação, instituído por $\mathrm{PaCO} 2$ menor que $32 \mathrm{mmHg}$ e alteração na contagem de células brancas, superior a $12.000 / \mathrm{cu} \mathrm{mm}$ ou menor que $4.000 / \mathrm{cu} \mathrm{mm}$; ou a presença de mais de $10 \%$ de neutrófilos imaturos. (BONE et al., 1992) Fica claro a importância de uma padronização mundial destas 
definições e métodos de identificação, "uma vez que órgãos de influência, como a Organização Mundial da Saúde, não se posicionam quanto ao endossamento de qualquer diretriz, deixando a critério de cada estabelecimento o endossamento ou não das diretrizes publicadas, dificultando, assim, a coleta de dados coesivos para a atualização dos parâmetros" (SATRIANO, 2017).

Em 1992 foi realizada a primeira conferência, chamada de Sepsis-1, que veio mudar as definições e diretrizes para a identificação de sepse e choque séptico, com o objetivo de melhorar o diagnóstico de pacientes com a enfermidade, levando à uma intervenção terapêutica precoce e a um melhor prognóstico (JAPARI et al, 1992). Baseado na evidência de que as definições de Sepsis-1 não promovem, adequadamente, o estabelecimento de um estadiamento de risco do paciente com sepse em 2001, foi realizada a segunda conferência de consenso para a atualização das definições, a Sepsis-2 (GRANJA; PÓVOA, 2015). Até o momento foram realizadas três grandes conferências para atualização dos conceitos envolvidos: a Sepsis-1 (1992), Sepsis-2 (2001), e Sepsis-3 (2016). As conferências têm como objetivo padronizar as definições e termos relacionados a sepse, como uma forma de dinamizar e agilizar o processo de diagnóstico e tratamento (MATOS; VICTORINO, 2004).

Recentemente ocorreu a conferência da Sociedade Europeia de Medicina de Cuidados Críticos (ESICM) e da Sociedade de Medicina de Cuidados Críticos (SCCM) na qual publicaram as novas definições de sepse (SEPSE-3). Estas, por sua vez, não foram baseadas apenas em opiniões de especialistas, mas sim embasadas em revisões sistemáticas bem como em análises de dados empíricos (SANTOS, et al 2017). Defende-se que Sepse é a presença de uma disfunção orgânica ameaçadora a vida secundária a resposta desregulada do organismo a infecção. Mediante esse novo conceito, todos os casos de sepse são graves, abolindo o termo "sepse grave" (SANTOS, et al 2017). Após a conferência de 2016 o termo "sepse grave" foi eliminado, uma vez que todos os casos de sepse devem ser vistos como grave. A nova definição ampla de sepse ficou definida como "presença de disfunção orgânica ameaçadora à vida secundária à resposta desregulada do organismo à infecção", 
sendo o seu diagnóstico clínico não mais baseado nos critérios da SIRS e sim no preenchimento de 2 ou mais pontos no escore SOFA (CARNEIRO; PÓVOA; GOMES, 2017).

Atualmente, para a confirmação do quadro clínico do paciente, com o objetivo de agilizar o diagnóstico, foi desenvolvido um sistema de score chamado de SOFA (Sequential Organ Faliure Assessment Score). É uma ferramenta usada na beira do leito para identificar pacientes com suspeita de infecção que estão sob maior risco de mortalidade (SINGER et al, 2016). Foi criado a partir do Sepsis-3 um parâmetro simplificado do score SOFA: o"quick SOFA" ou qSOFA. Este parâmetro inclui: (1) Frequência respiratória $\geq 22$ /minuto, (2) Estado mental alterado e (3) Pressão Arterial Sistólica $\leq 100 \mathrm{mmHg}$ e é de rápida análise. Se pelo menos dois dos critérios forem confirmados, considera-se alto risco de mortalidade (SINGER et al, 2016). SOFA é utilizado para o diagnóstico clínico da disfunção orgânica, sendo necessário dois ou mais pontos no score. Foi sugerido um score simplificado qSOFA utilizado apenas para triagem de pacientes graves. Ele se torna positivo quando o paciente apresenta dois de seus critérios (SANTOS, et al 2017).

Os critérios clínicos do qSOFA são: frequência respiratória maior ou igual 22 incursões por minuto, alteração do nível de consciência (segundo a Escala de Coma de Glasgow inferior a 15), pressão arterial sistólica menor ou igual a $100 \mathrm{mmHg}$. Estes são utilizados na beira do leito de forma rápida a fim de se identificar possíveis desfechos clínicos desfavoráveis (MACHADO et al, 2016). Este novo score trouxe variáveis antes negligenciadas como a elevação da frequência respiratória e diminuição do nível de consciência, que são marcadores de gravidade e mortalidade. Não devendo ser utilizado para diagnóstico de sepse (MACHADO et al, 2016). Segundo Machado et al, (2016) as novas definições de sepse apresentam como vantagens: uma melhor descrição de sepse como processo fisiológico; o fato da padronização ter sido baseada em dados disponíveis e não na opinião de especialistas; ela não exigir a presença da SIRS, que não é nem sensível e nem especifica para sepse; o abandono do termo "sepse grave", já que toda sepse é grave; a melhor definição de choque 
séptico; o fato do qSOFA levar em consideração o nível de consciência e a elevada freqüência respiratória.

Como desvantagens há a redução de sensibilidade para detectar casos que podem ter evolução desfavorável; o fato do escore não ser bem conhecido pelos profissionais de saúde que trabalham no pronto-socorro ou nas enfermarias e a sua aplicabilidade ser complexa, pois pode demandar o cálculo do SOFA nos dias subsequentes para verificar se o paciente cumpre os critérios estritos bem como pode-se necessitar de exames laboratoriais adicionais; há, ainda e a desvalorização de hiperlactatemia isolada na fase aguda de infecção como uma disfunção orgânica metabólica; a nova definição de choque séptico, na qual se exige hiperlactatemia como componente obrigatório para a definição, diferente dos consensos Sepsis 1 e Sepsis 2, nos quais a simples presença de hipotensão refratária à administração de fluidos era considerada choque (SATRIANO, 2017).

A definição de choque séptico também sofreu modificação, assim ficou definido como: "um subgrupo de pacientes com sepse que apresentam acentuadas anormalidades circulatória, celulares e metabólicas, associadas com maior risco de morte do que a sepse isoladamente" e tendo como critério a "necessidade de vasopressor para manter uma pressão arterial média acima de $65 \mathrm{mmHg}$ após a infusão adequada de fluidos, associada a nível sérico de lactato acima de $2 \mathrm{mmol} / \mathrm{L}$ " (MACHADO et al., 2016). Segundo Machado (2016), tanto a Associação de Medicina Intensiva Brasileira (AMIB) quanto o Instituto Latino Americano de Sepse (ILAS) acreditam que o novo critério para sepse é apropriado. Porém, elas consideram que não será viável o uso de critérios estritos para definir sepse em programas de melhoria de qualidade no Brasil. O autor defende que alguns aspectos das novas definições não seriam aplicáveis em termos práticos na beira do leito sem o risco de uma redução da sensibilidade e de retardo no reconhecimento da sepse (SATRIANO, 2017).

\section{CONSIDERAÇÕES FINAIS}

Para a prática dos novos critérios é necessária a atualização dos profissionais de saúde afim de se identificar e tratar a sepse em tempo oportuno, garantindo, assim 
uma adequada assistência à saúde bem como resultados melhores, para, com isso haver uma diminuição expressiva dos óbitos causados por essa condição (SANTOS, et al 2017).

\section{REFERÊNCIAS}

BATISTA, R. S. et al. Atualidades proteômicas na sepse. Rev. Assoc. Med. Bras, vol. 58, n.3, 2012.

BARACHO, N. C. V. et al. Fatores de risco associados à mortalidade em pacientes com sepse grave e choque séptico na unidade de terapia intensiva de um hospital escola do sul de Minas Gerais. Revista Ciências em Saúde v. 1, n. 1, 2011.

BONE, R. C. ACCP/SCCM consensus conference: Definitions for Sepsis and Organ Failure and Guidelines for the use of innovative therapies in Sepsis. Chest Journal, Glenview, v. 101, n. 6, p. 1644-1655, 1992.

CARNEIRO, A. H.; PÓVOA, P.; GOMES, J. A. Dear Sepsis-3, we are sorry to say that we don't like you. Revista Brasileira de Terapia Intensiva, São Paulo, v. 29, n. 1, p. 4-8, 2017.

GRANJA, C.; PÓVOA, P. PIRO. Estratificação da sepse: realidade ou miragem? Revista Brasileira de Terapia Intensiva. São Paulo, v. 27, n. 3, p. 196-198, 2015.

ILAS - Instituto Latino-Americano para Estudos da Sepse. Sepse: um problema de saúde pública. Instituto Latino-Americano para Estudos da Sepse. Brasília, CFM, 2015.

JAPARI, H. S. et al. Sepsis and septic shock: a review for clinicians. Pediatric Infectious Disease Journal, Philadelphia. v. 11, n. 9, p. 739-749, 1992.

JUNIOR, J. A. L. S. et al. Sepse Brasil: Estudo Epidemiológico da Sepse em Unidades de Terapia Intensiva Brasileiras. Revista Brasileira de Terapia Intensiva, v. 18, n. 1, 2006. 
MATOS, G. F. J; VICTORINO, J. A. Critérios para o Diagnóstico de Sepse, Sepse grave e Choque Séptico. Revista Brasileira de Terapia Intensiva. São Paulo, v. 16, n. 2, p. 102-104, 2004.

MACHADO, F. R. et al. Chegando a um consenso: vantagens e desvantagens do Sepsis 3 considerando países de recursos limitados. Revista Brasileira Terapia Intensiva. São Paulo, v. 28, n. 4, p. 361-365, 2016.

MOURA, J. M. et al. Diagnóstico de sepse em pacientes após internação em unidade de terapia intensiva. Arq. Ciênc. Saúde, 2017.

SATRIANO, P. de. M. Evolução das diretrizes internacionais de definição e identificação da sepse e choque séptico. 2017. 22 f. Monografia (Graduação) Faculdade de Ciências da Educação e Saúde, Centro Universitário de Brasília. Brasília, 2017.

SANTOS, H. A. et al. As novas diretrizes de sepse e a atuação da enfermagem. In: INTERNATIONAL NURSING CONGRESS Theme: Good practices of nursing representations in the construction of society, 2017.

SHORR, A. F. et al. Economic implications of an evidence-based sepsis protocol: can we improve outcomes and lower costs? Crit Care Med, v. 35, n. 5, p. 1257-1262, 2007.

SINGER, M. et al. The third International Consensus Definitions for Sepsis and Septic Shock (Sepsis-3). Journal of the American Medical Association. New York, v. 315, n. 8, p. 801- 810, 2016.

Enviado: Maio, 2019.

Aprovado: Junho, 2019. 